\title{
Animal Health Research Reviews \\ Canine vector-borne disease: mapping and the accuracy of forecasting using big data from the veterinary community \\ cambridge.org/ahr
}

\section{Review}

${ }^{*}$ Current address: School of Community Health Sciences, University of Nevada, Reno NV, USA

tCurrent address: Nicholas School of the Environment, Duke University, Durham NC, USA

Cite this article: Self SCW et al (2019). Canine vector-borne disease: mapping and the accuracy of forecasting using big data from the veterinary community. Animal Health Research Reviews 20, 47-60. https://doi.org/10.1017/ S1466252319000045

Received: 20 August 2018

Revised: 23 April 2019

Accepted: 9 July 2019

First published online: 26 September 2019

\section{Key words:}

Anaplasma; canine vector-borne disease; Ehrlichia; epidemiology; forecast; heartworm; Lyme; One Health

Author for correspondence:

Jenna R. Gettings, E-mail: jrgettings@gmail. com

\section{Stella C. W. Self ${ }^{1}$, Yan Liu ${ }^{1, \star}$, Shila K. Nordone ${ }^{2, \dagger, ~ M i c h a e l ~ J . ~ Y a b s l e y ~}{ }^{3,4}$, Heather S. Walden ${ }^{5}$, Robert B. Lund ${ }^{1}$, Dwight D. Bowman ${ }^{6}$, Christopher Carpenter ${ }^{7}$, Christopher S. McMahan ${ }^{1}$ and Jenna R. Gettings ${ }^{1,3}$ (D)}

\footnotetext{
${ }^{1}$ School of Mathematical and Statistical Sciences, Clemson University, Clemson, SC, USA; ${ }^{2}$ Department of Molecular and Biomedical Sciences, Comparative Medicine Institute, College of Veterinary Medicine, North Carolina State University, Raleigh, NC, USA; ${ }^{3}$ Southeastern Cooperative Wildlife Disease Study, Department of Population Health, College of Veterinary Medicine, The University of Georgia, Athens, GA, USA; ${ }^{4}$ Warnell School of Forestry and Natural Resources, The University of Georgia, Athens, GA, USA; ${ }^{5}$ Department of Comparative Diagnostic and Population Medicine, College of Veterinary Medicine, University of Florida, Gainesville, Florida, USA; ${ }^{6}$ College of Veterinary Medicine, Cornell University, Ithaca, NY, USA and ${ }^{7}$ Companion Animal Parasite Council, Salem, OR, USA
}

\begin{abstract}
Diagnosis, treatment, and prevention of vector-borne disease (VBD) in pets is one cornerstone of companion animal practices. Veterinarians are facing new challenges associated with the emergence, reemergence, and rising incidence of VBD, including heartworm disease, Lyme disease, anaplasmosis, and ehrlichiosis. Increases in the observed prevalence of these diseases have been attributed to a multitude of factors, including diagnostic tests with improved sensitivity, expanded annual testing practices, climatologic and ecological changes enhancing vector survival and expansion, emergence or recognition of novel pathogens, and increased movement of pets as travel companions. Veterinarians have the additional responsibility of providing information about zoonotic pathogen transmission from pets, especially to vulnerable human populations: the immunocompromised, children, and the elderly. Hindering efforts to protect pets and people is the dynamic and ever-changing nature of VBD prevalence and distribution. To address this deficit in understanding, the Companion Animal Parasite Council (CAPC) began efforts to annually forecast VBD prevalence in 2011. These forecasts provide veterinarians and pet owners with expected disease prevalence in advance of potential changes. This review summarizes the fidelity of VBD forecasts and illustrates the practical use of CAPC pathogen prevalence maps and forecast data in the practice of veterinary medicine and client education.
\end{abstract}

(c) The Author(s) 2019. This is an Open Access article, distributed under the terms of the Creative Commons Attribution licence (http:// creativecommons.org/licenses/by/4.0/), which permits unrestricted re-use, distribution, and reproduction in any medium, provided the original work is properly cited.

\section{CAMBRIDGE} UNIVERSITY PRESS

\section{Introduction}

Over the last several decades, health care providers, scientists, and public health officials have observed a disconcerting trend in infectious diseases: the emergence and reemergence of numerous pathogens. Emerging pathogens are those that appear in a new population or those that display increasing incidence in an existing population (Woolhouse and Gowtage-Sequeria, 2005). Among infectious pathogens, zoonotic pathogens are roughly 1.9 times more likely to be emerging than non-zoonotic pathogens; vector-borne pathogens are about 2.3 times more likely to be emerging than non-vector-borne pathogens (Taylor et al., 2001). A number of factors are thought to contribute to the increasing incidence of diseases caused by vector-borne pathogens. These include (1) warmer winters and other temperature and humidity alterations that are occurring as a result of climate change (Eisen et al., 2015), (2) suburbanization, which brings people, wildlife, domestic animals, and pathogens together, (3) an increase in white-tailed deer or other wildlife species that support ticks and/or serve as reservoirs of infection, (4) migratory birds that carry ticks or pathogens to new areas, (5) the modern emphasis on the preservation of open space and the replanting of trees, and (6) the reduced use of insecticides (Loh et al., 2015). These factors are dynamic; as a result, it is expected that the incidence, prevalence, and spatial distribution of vector-borne disease (VBD) will continue to change.

This changing nature of VBDs makes the surveillance of disease incidence and prevalence an integral part of prevention and control. In the United States (US), monitoring human VBDs is generally conducted through formal state and national surveillance systems (National Notifiable Diseases Surveillance System (NNDSS), Centers for Disease Control and Prevention), giving health care providers invaluable information to direct interventions, 
preventative care measures, and public education. In contrast, although US veterinarians observe several of the same VBD agents in domestic dogs (including, but not limited to, Borrelia burgdorferi, Anaplasma phagocytophilum, Ehrlichia chaffeensis, and E. ewingii), none of these pathogens (in dogs) are currently nationally notifiable and state-level requirements vary. In the absence of a formal surveillance system, many veterinarians rely on available animal health industry data to assess the infection prevalence. Since 2011, the Companion Animal Parasite Council (CAPC) has worked collaboratively with IDEXX Laboratories, Inc. and Antech Diagnostics to assemble a diagnostic test result database from practicing veterinarians. From these data, CAPC provides prevalence (proportion of animals that test positive in a given place and time period; seroprevalence is defined in this manuscript as the proportion of animals with positive antibody tests) maps at the county, state, and national levels for both zoonotic and non-zoonotic pathogens of companion animals: B. burgdorferi, Ehrlichia spp., Anaplasma spp., Dirofilaria immitis, and Giardia spp. for dogs; and D. immitis, Giardia spp., Feline Leukemia Virus, and Feline Immunodeficiency Virus for cats. Results from centrifugal fecal flotation examinations (roundworms, hookworms, and whipworms) for both dogs and cats are also reported (Companion Animal Parasite Council, 2018). The prevalence maps provide local and regional surveillance data to veterinarians and scientists alike, facilitating evidence-based medicine and informing research. The known association between canine and human VBD (Mead, 2015; Liu et al., 2019) highlights the importance of these maps in the One Health framework. Of the above listed pathogens, B. burgdorferi, A. phagocytophilum, E. chaffeensis and E. ewingii account for the highest number of reported cases of human tick-borne disease in the US (Rosenberg et al., 2018). Surveillance of these pathogens in pets helps protect both animals and people.

Of all the aforementioned pathogens for which prevalence data are available, four canine pathogens have datasets that are robust enough to perform annual forecasting: D. immitis, Anaplasma spp., Ehrlichia spp., and B. burgdorferi. The forecasting methods use both the historical prevalence and other environmental factors theorized to influence vector survival and exposure to VBD pathogens (Bowman et al., 2016; Watson et al., 2017; Liu et al., $2017 a, 2017 b$ ). Monthly and annual updates to the prevalence and forecast maps, respectively, are imperative to adequately understand exposure risk. Similar to prevalence maps, all forecast maps are free to the general public, veterinarians, and scientists at www.petsandparasites.org/parasite-prevalence-maps or https:// capcvet.org/maps.

This review summarizes the prevalence maps and the modeling used to create annual forecast maps for B. burgdorferi, Ehrlichia spp., Anaplasma spp., and D. immitis and extends original discussions to include a description of the development and fidelity of annual forecast maps. Importantly, the goal of this review is to describe the use of forecast maps to the practicing veterinarian for the purpose of client education and evidencebased decision-making. A discussion of the importance of these forecast maps, their intended use, and how they can aid the practicing veterinarian in preventative medicine is presented.

\section{Parasite prevalence maps}

CAPC receives monthly data from IDEXX Laboratories, Inc. and Antech Diagnostics and presents it to the public in the form of interactive maps (Companion Animal Parasite Council, 2018).
The maps display the proportion of animals that test positive among all animals tested within a given county for a given period (month or year). Maps are available for D. immitis (antigen), Ehrlichia spp., Anaplasma spp., and B. burgdorferi exposure and infections in dogs; D. immitis (antigen and antibody), Feline Immunodeficiency Virus (antigen), and Feline Leukemia Virus (antibody) infections in cats; and intestinal parasites in both dogs and cats, including Toxocara spp., Ancylostoma spp., Trichuris spp., and Giardia spp. Data are available for the US and Canada and are updated monthly from January 2012 through the present. Importantly, data can be viewed for the annual or monthly periods at the national, state/province, or county/ county-equivalent level (within the US), allowing veterinarians to provide local, regional, and national expertise on pathogen prevalence for their clients. These data are intended to describe the prevalence of parasites in a given locality, but as with any survey, the results can be influenced by a number of factors, including the number of pets tested, the history of the pets prior to testing, the reason the pets were tested, and the assays used. Understanding each of these factors is important when inferring the geographic distribution of these disease agents. Factors that can influence data interpretation are described at https://www. capcvet.org/articles/under-standing-the-maps-key-factors-thatinfluence-the-results/. Details on the pathology, diagnostics, and epidemiology of these pathogens are beyond the scope of this review and many excellent references are available for more information (Little, 2010; Little et al., 2010; Greene, 2012; Allison and Little, 2013; Bowman, 2014; Maggi et al., 2014; Qurollo et al., 2014; Sykes, 2014; Littman et al., 2018).

To ensure that prevalence estimates are not unduly influenced by test quality, data used for the prevalence maps meet stringent inclusion criteria. Antibody tests for the tick-borne disease agents (B. burgdorferi, Ehrlichia spp., and Anaplasma spp.) must have a minimum sensitivity of $90 \%$ and a minimum specificity of $90 \%$. CAPC also requires that the in-clinic tests used by veterinarians to acquire data be externally validated via approval by the US Department of Agriculture (USDA). Information regarding USDA regulation of diagnostic tests can be found at http://www. aphis.usda.gov/animal_health/vet_biologics/publications/pel_4_7.pdf. For heartworm infection, a minimum sensitivity of $90 \%$ and a minimum specificity of $90 \%$ are required for all antigen tests. Because the sensitivity of some heartworm antigen tests declines at lower worm burdens, the $90 \%$ sensitivity requirement applies only when three or more adult female worms are present (Atkins, 2003). Similar to antibody test inclusion criteria, CAPC requires that heartworm antigen tests be approved by the USDA. Intestinal parasite data are acquired via fecal flotation. The roundworm, hookworm, and whipworm data are acquired via centrifugal fecal flotation. Because sensitivity and specificity are variable, all fecal results that follow the procedures, which include centrifugation and minimum sample size of $1 \mathrm{~g}$, are accepted. The resultant data should be interpreted under these limitations.

\section{Forecast maps}

CAPC uses historical VBD prevalence data along with established climatological and ecological drivers of disease to construct annual forecast maps that display the expected prevalence of seropositive animals for the upcoming year for each of the four VBD pathogens described above. Annual forecast efforts began in 2011, with the first forecast based on the Bayesian spatio-temporal 
Poisson regression model (described below) delivered in 2016. Additional details are available in Bowman et al. (2016), Liu et al. $(2017 a, 2017 b)$, and Watson et al. (2017).

\section{Data}

The data used to forecast are the same as those used in the prevalence maps. Data are obtained as aggregated counts of positive tests or total tests by county and month. These data can be seen in each of the raw prevalence maps (Figs. 1a, 2a, 3a, and 4a). Counts of positive tests and total tests performed in a given county from 2012 through 2018 were summed. The proportion of all positive tests to total tests in a given county is the overall prevalence for that time period. Heartworm antigen test results are obtained from IDEXX Laboratories, Inc. (SNAP ${ }^{\circledR}$ Heartworm RT Test, SNAP ${ }^{\circledR} 4 \mathrm{Dx}^{\circledast}$ Plus Test, and PetChek ${ }^{\circledast}$ Heartworm PF Antigen Test) and Antech Diagnostics (DiroCHEK ${ }^{\oplus}$ Heartworm Antigen Test and AccuPlex4 heartworm antigen detection assay). The remaining data are obtained from IDEXX (SNAP ${ }^{\circledast} 4 \mathrm{Dx}^{\oplus}$ Plus Test). Data are collected both from tests performed at the reference laboratories and from clinics that are connected to the laboratories via clinic practice management software. No measure of uncertainty is given for any test. Travel, disease, and testing histories of the dogs are also not available. Roughly half of the 3109 counties, boroughs, parishes, and other county-equivalent areas in the US do not report any tests in a given year; in contrast, some highly populated counties report thousands of tests annually. More details on the data can be found in Bowman et al. (2016); Liu et al. (2017a, 2017b), and Watson et al. (2017).

\section{Covariates}

The statistical model described in the next section uses many putative risk factors to improve the annual prevalence forecasts. Eight risk factors (covariates or predictor variables) are considered: annual mean temperature, annual mean precipitation, annual mean relative humidity, elevation, percent forest coverage, percent water coverage, population density, and median household income. The listed factors were previously selected based on similar models. See Wang et al. (2014) for more information. Most of these factors are available to a county spatial resolution. In addition to these eight factors, the heartworm forecast also uses the presence/absence of eight mosquito species: Aedes albopictus, A. aegypti, A. canadensis A. sierrensis, A. trivittatus, Anopheles punctipennis, Anopheles quadrimaculatus, and Culex quinquefasciatus.

Data for the proposed risk factors were obtained from a variety of sources. Sources for all forecasts made up to 2017 are discussed in Wang et al. (2014). The forecast models are updated annually. As such, covariate data sources are periodically updated. This occurs for multiple reasons: prior sources become unavailable, data accuracy or spatio-temporal resolution is improved, new knowledge is gained about potential risk factors, or data acquisition becomes easier. In an effort to provide the most reliable and up to date information, covariate sources were updated for our 2018 and 2019 forecasts presented below.

As one would expect, changing covariate sources can change the significance level of the individual covariates and the forecast. However, the changes are usually not large. For clarity, the significance of factors based on 2011-2015 data will be reported. These years are chosen to be consistent with the data used in publications for each pathogen: B. burgdorferi (Watson et al., 2017), Anaplasma spp. (Liu et al., 2017b), Erhlichia spp. (Liu et al., 2017a), and heartworm (Bowman et al., 2016). These studies used the most recent data available at the time of publication. For B. burgdorferi and Anaplasma spp., elevation, percent forest coverage, and percent water coverage are positively related to prevalence; annual temperature and population density are negatively related to prevalence; annual precipitation, annual relative humidity, and median household income are statistically insignificant. For Ehrichlia spp., annual temperature, percent forest coverage, and percent water coverage are positively related to prevalence; population density and median household income are negatively related to prevalence; and annual precipitation, annual relative humidity, and elevation are insignificant. For heartworm, annual temperature, annual relative humidity, and percent forest coverage are positively related to prevalence; population density, median household income, and presence of $A$. albopictus are negatively related to prevalence; and annual precipitation, elevation, percent water coverage, and presence of $A$. aegypti, A. canadensis A. sierrensis, A. trivittatus, A. punctipennis, A. quadrimaculatus, and $C$. quinquefasciatus are insignificant.

\section{Forecast model}

This technical section describes the statistical methods used in making the forecasts. For notation, let $Y_{\text {st }}$ and $n_{\text {st }}$ denote the number of positive and total tests reported in county $s$ during year $t$, respectively. In general, counties that are geographically close to each other often report a similar prevalence. Similarly, a fixed county tends to report a similar disease prevalence in consecutive years. These phenomena entail positive spatio-temporal correlation and need to be accounted for in an accurate statistical analysis (Besag, 1974; Martínez-Beneito et al., 2008; López-Quilez and Munoz, 2009; Banerjee et al., 2014).

Our forecast model is Bayesian and accounts for spatiotemporal dependence using random effects as described below. In the model, the number of positive tests in each county and year is assumed to conditionally follow a Poisson distribution, which is a common choice for modeling count data (Martínez-Beneito et al., 2008; López-Quilez and Munoz, 2009). Specifically, $Y_{\text {st }}$ is assumed to be statistically distributed as

$$
Y_{\mathrm{st}} \mid n_{\mathrm{st}}, p_{\mathrm{st}} \sim \operatorname{Poisson}\left(n_{\mathrm{st}} p_{\mathrm{st}}\right)
$$

where

$$
\log \left(p_{\mathrm{st}}\right)=\beta_{0}+\sum_{k=1}^{p} \beta_{k} X_{k \mathrm{st}}+\xi_{\mathrm{st}}
$$

where $X_{k s t}$ is the $k$ th covariate at county $s$ and time $t, \beta_{k}$ is the corresponding regression coefficient, $p_{\text {st }}$ is the prevalence, and $\xi_{\text {st }}$ are the spatio-temporal random effects. Equation (1) posits that the number of positive tests $Y_{\text {st }}$, given the number of tests conducted $n_{\text {st }}$ and the prevalence $p_{\text {st }}$, follows a Poisson distribution with mean $n_{\text {st }} p_{\text {st }}$.

There are many model choices for the spatio-temporal random effects. Perhaps the most popular for spatial data is the conditional autoregression (CAR) model (Banerjee et al., 2014), which is used here. Spatial and temporal dependence is modeled by embedding a CAR model in the following first-order temporal 


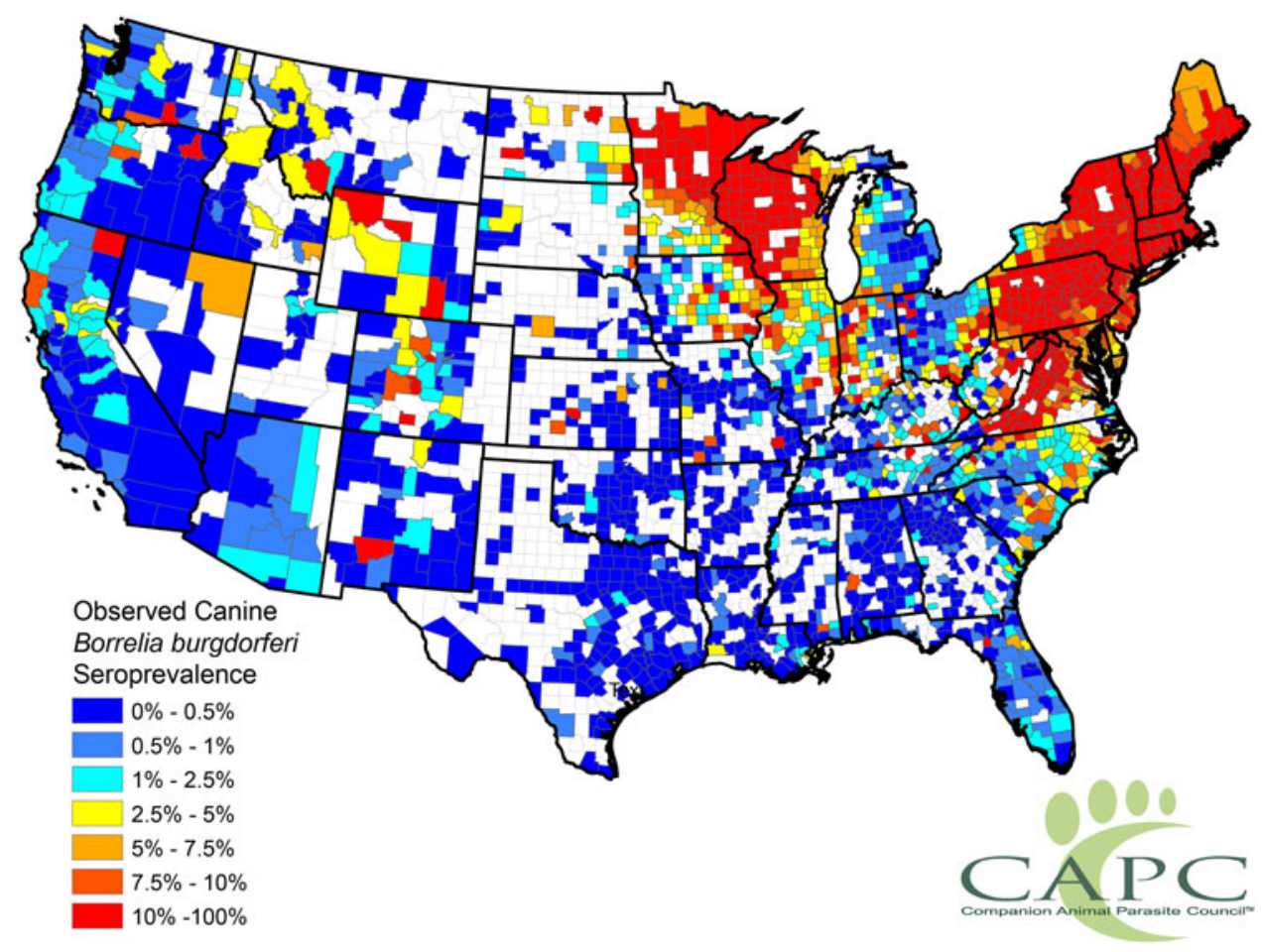

(A)

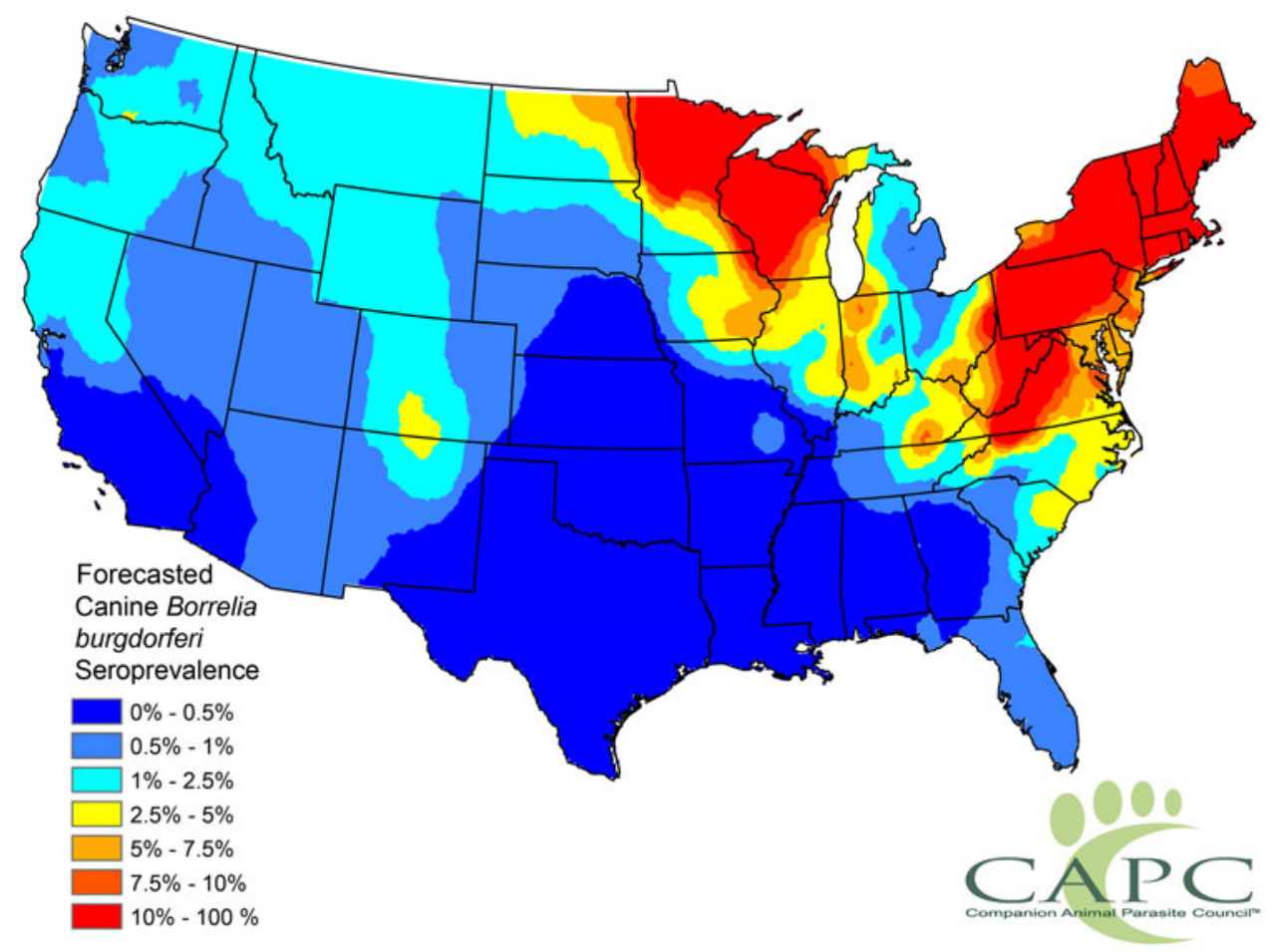

(B)

Fig. 1. Raw canine Borrelia burgdorferi prevalence aggregated by county from 2012 through 2018 and forecasted B. burgdorferi prevalence for 2019 . Counts for positive tests and for total tests performed in a given county from 2012 through 2018 were summed. The proportions of all positive tests to total tests in a given county are shown in (a). The expected seroprevalence of B. burgdorferi in 2019 is shown in (b). 


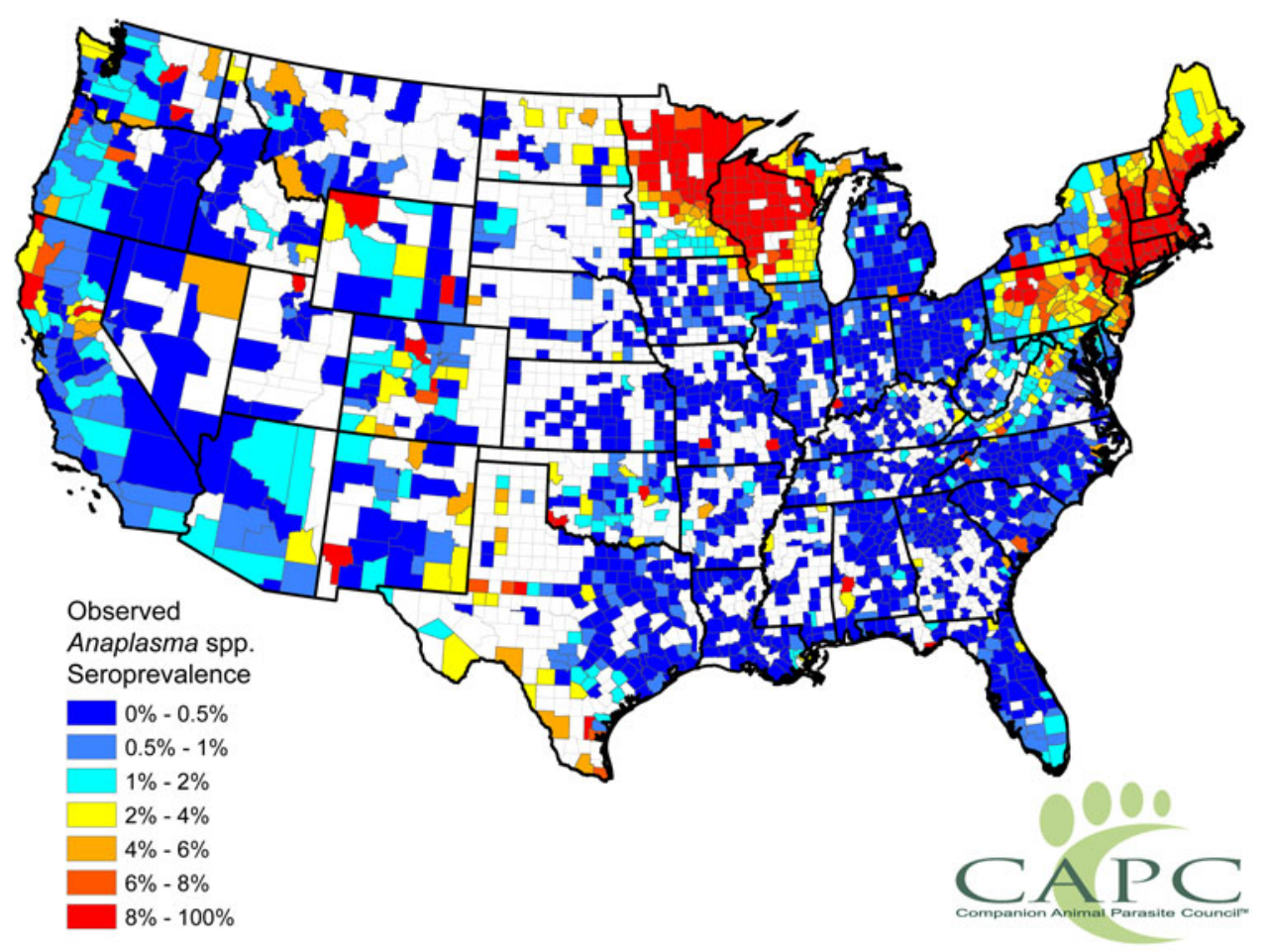

(A)

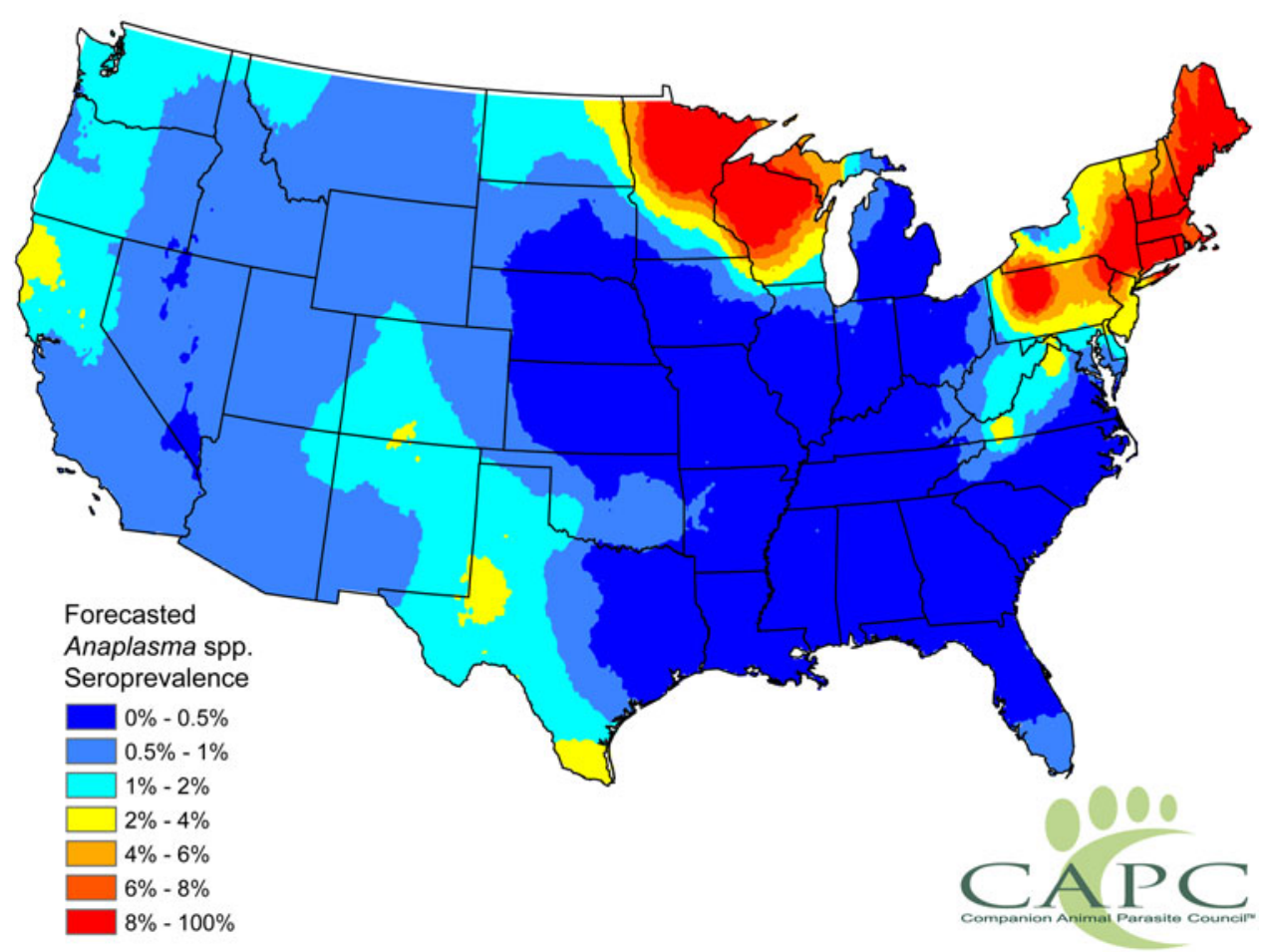

(B)

Fig. 2. Raw canine Anaplasma species prevalence aggregated by county from 2012 through 2018, and forecasted Anaplasma spp. prevalence for 2019. Counts for positive tests and for total tests performed in a given county from 2012 through 2018 were summed. The proportions of all positive tests to total tests in a given county are shown in (a). The expected seroprevalence of Anaplasma spp. in 2019 is shown in (b). 


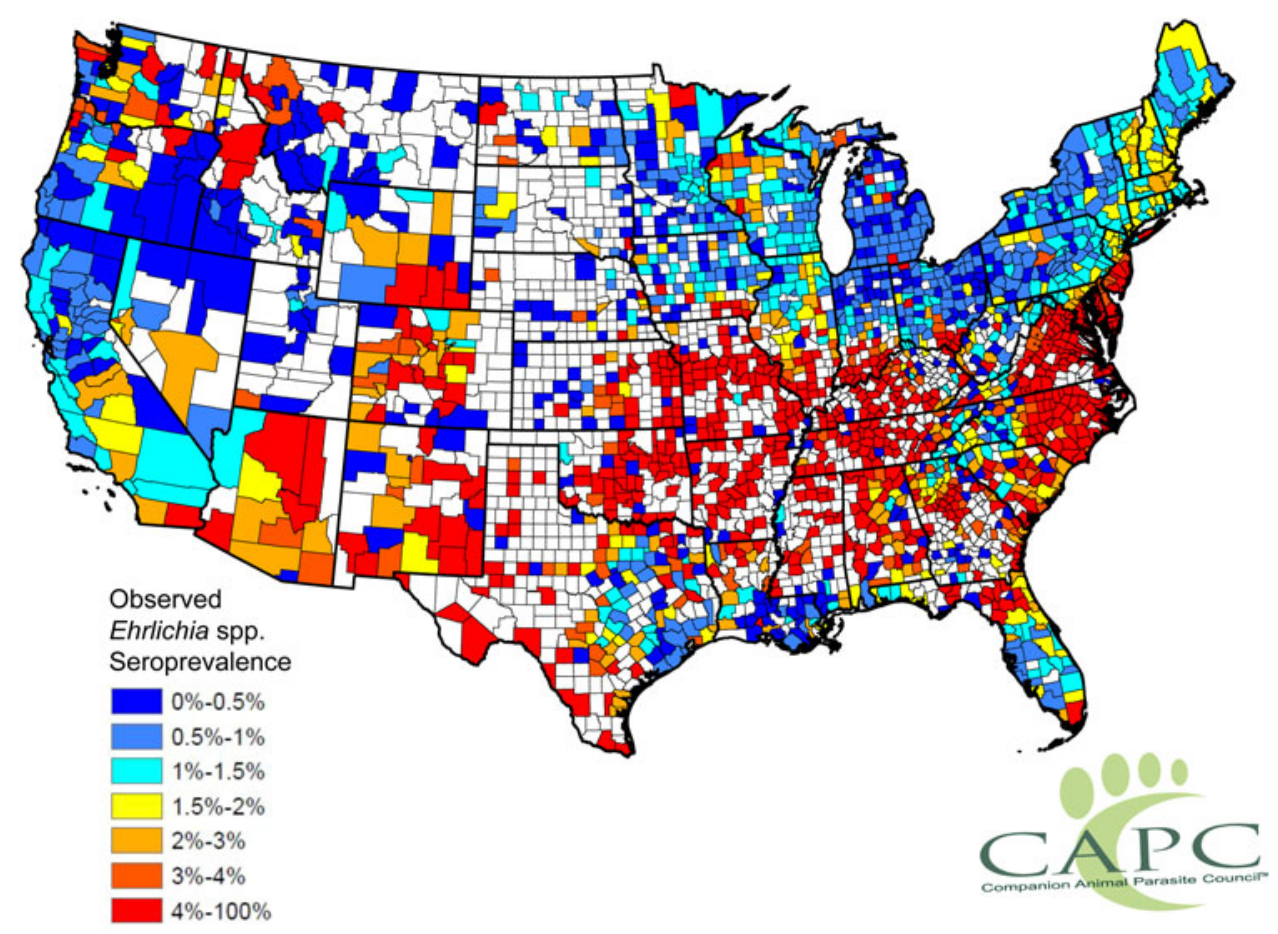

(A)

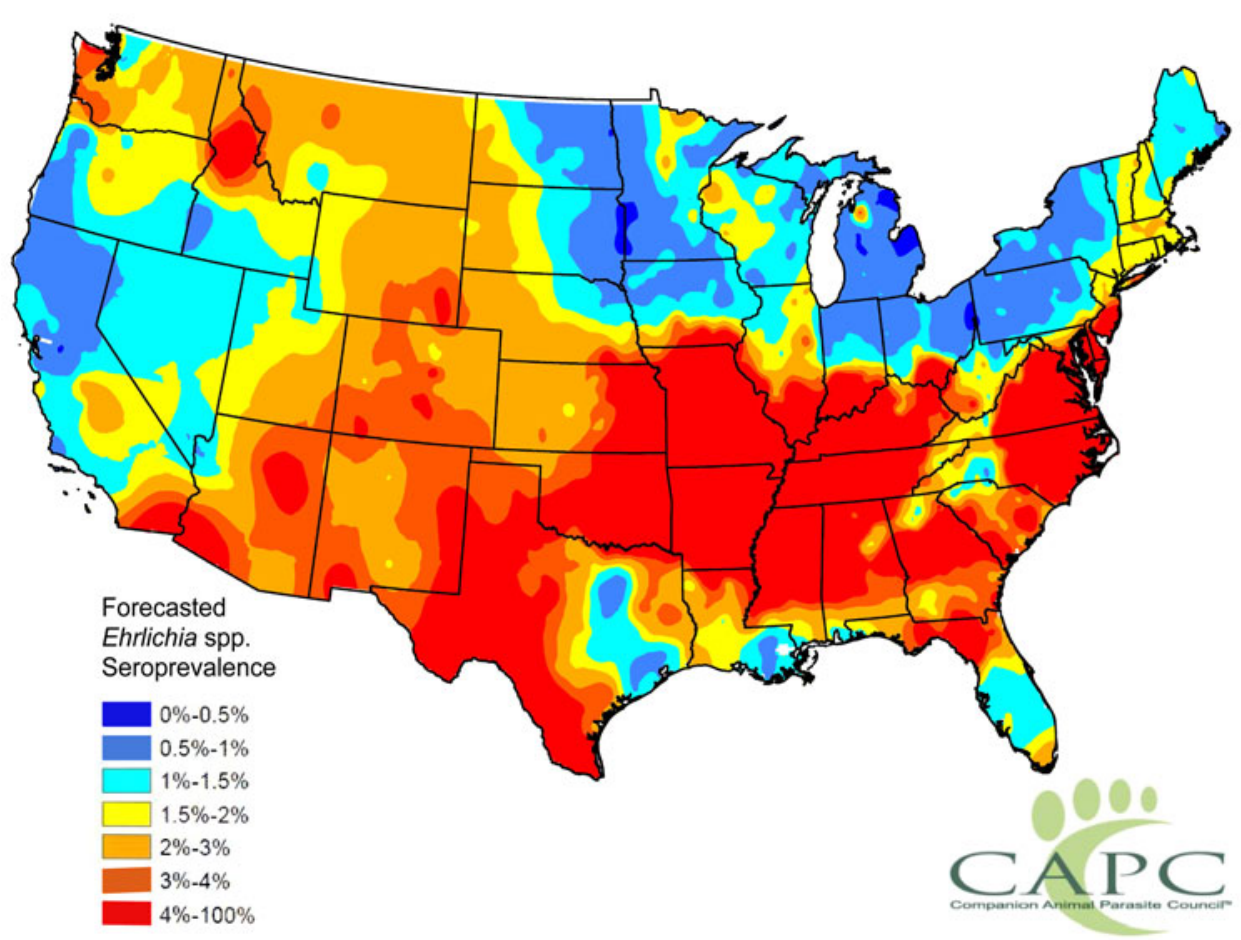

(B)

Fig. 3. Raw canine Ehrlichia species prevalence aggregated by county from 2012 through 2018, and forecasted Ehrlichia spp. prevalence for 2019 . Counts for positive tests and for total tests performed in a given county from 2012 through 2018 were summed. The proportions of all positive tests to total tests in a given county are shown in (a). The expected seroprevalence of Ehrlichia spp. in 2019 is shown in (b). 


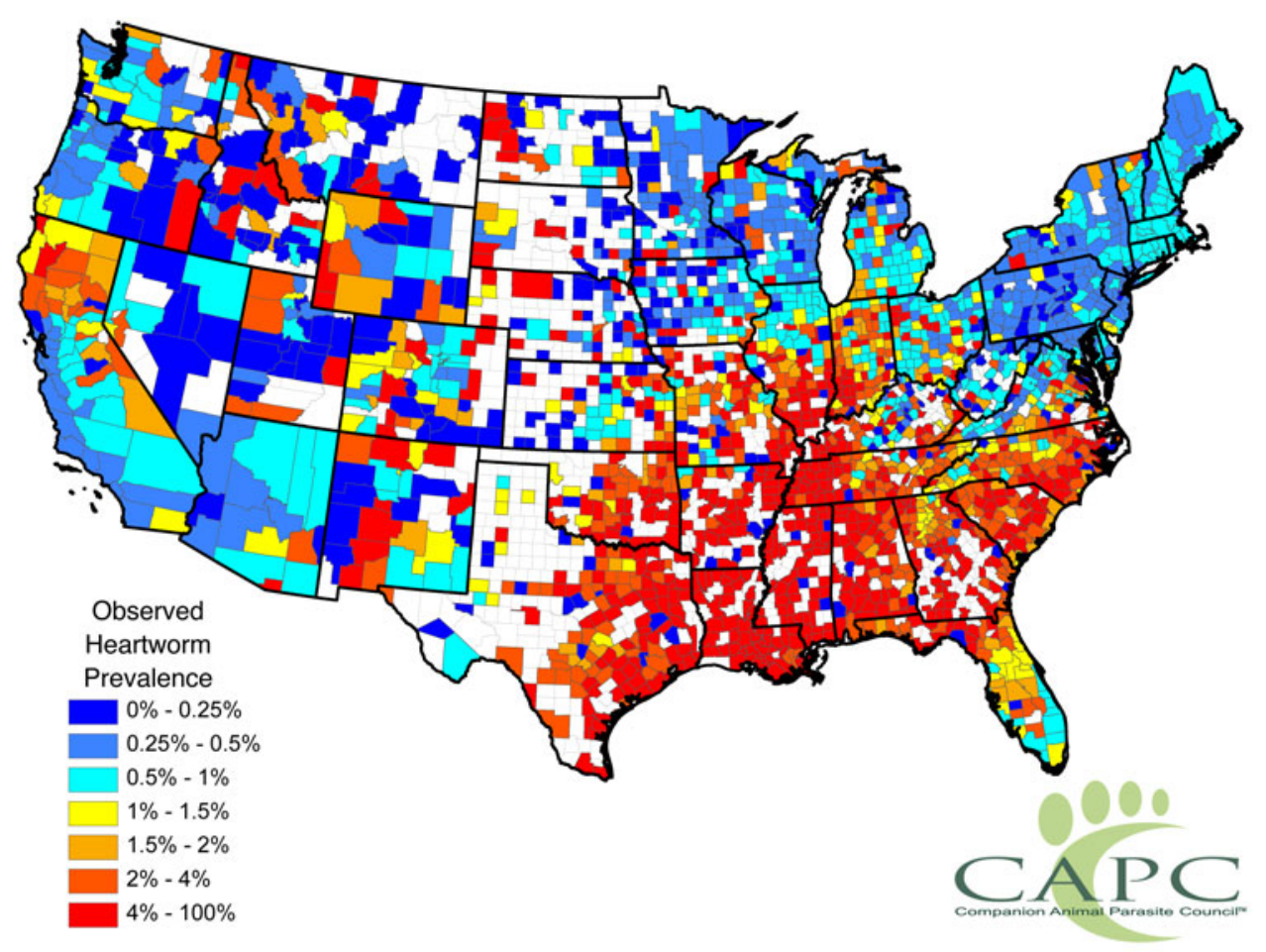

(A)

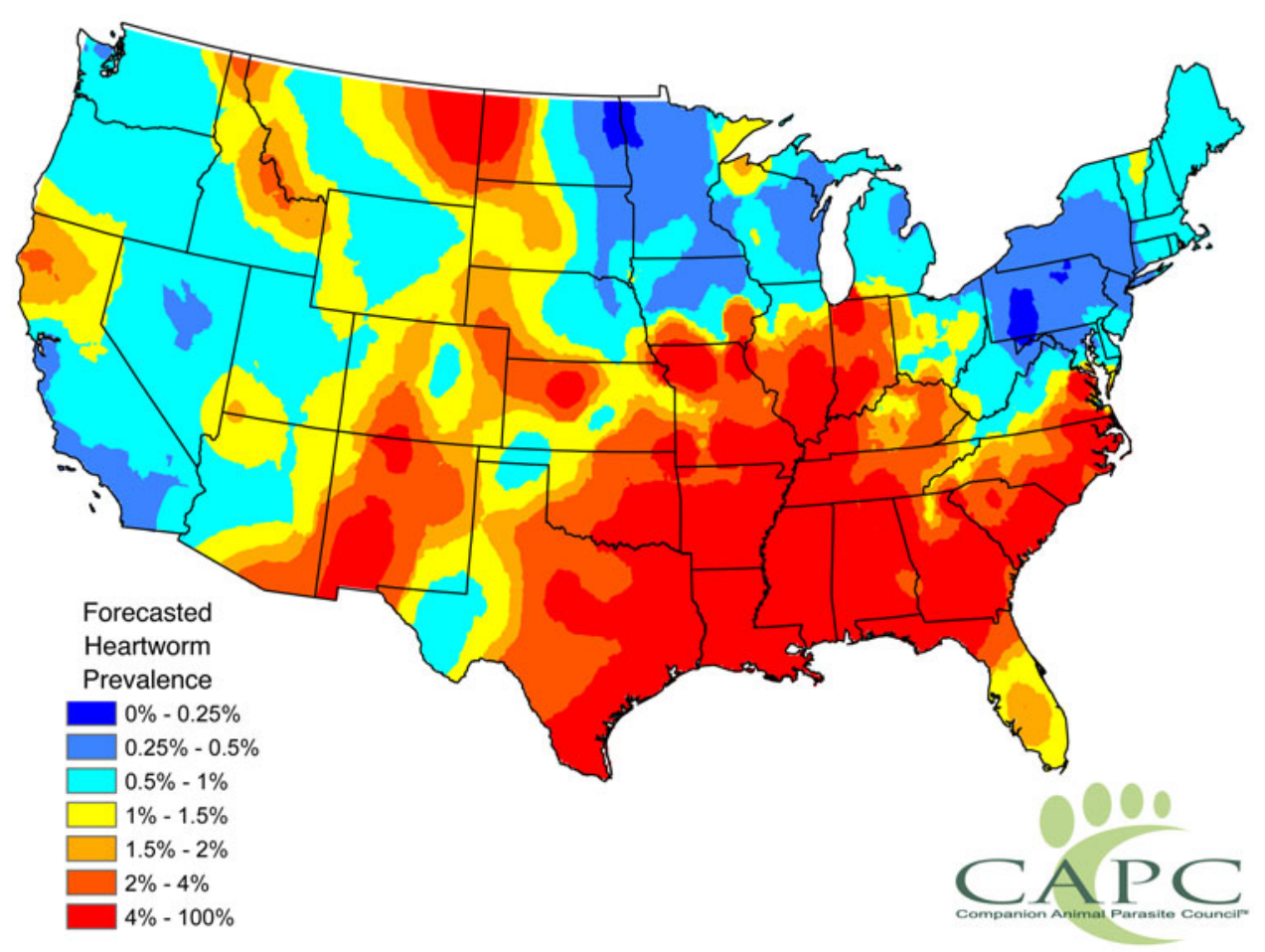

(B)

Fig. 4. Raw canine heartworm prevalence aggregated by county from 2012 through 2018 , and forecasted heartworm prevalence for 2019 . Counts for positive tests and for total tests performed in a given county from 2012 through 2018 were summed. The proportions of all positive tests to total tests in a given county are shown in (a). The expected prevalence of heartworm in 2019 is shown in (b). 
autoregressive scheme:

$$
\begin{aligned}
\xi_{1} & =\phi_{1} \\
\xi_{t} & =\varphi \xi_{t-1}+\phi_{t}, \text { for } t \geq 2 \\
\boldsymbol{\phi}_{t} & \sim \operatorname{CAR}\left(\tau^{2}, \rho\right), \text { for } t \geq 1,
\end{aligned}
$$

where $\boldsymbol{\xi}_{t}=\left(\xi_{1 t}, \ldots, \xi_{\mathrm{St}}\right)$, and where $\boldsymbol{\phi}_{t}=\left(\phi_{1 t}, \ldots, \phi_{\mathrm{St}}\right)$. Timedependence is accounted for with a first-order temporal autoregression, which is commonly used to model temporal data (Brockwell and Davis, 2002). The parameter $\varphi$ lies between -1 and 1 and controls the level of temporal correlation between consecutive years.

Under our spatio-temporal model, the $\boldsymbol{\phi}_{t}$ 's are independent and identically distributed random vectors in time $t$ whose distribution follows a CAR model (Besag, 1974; Banerjee et al., 2014). Typically, CAR models are defined by specifying a univariate conditional distribution for the random effect at each county $s$. The mean and variance of this conditional distribution depend on the spatial relationship between the counties. Our forecast model uses the CAR model variant proposed in Besag (1974):

$$
\phi_{\text {st }} \mid \boldsymbol{\phi}_{-s t}, \tau^{2}, \rho \sim \operatorname{Normal}\left(\frac{\rho}{d_{s}} \sum_{s^{\prime} \neq s} w_{s^{\prime} s} \phi_{s^{\prime}}, \frac{\tau^{2}}{d_{s}}\right), \text { for all } s .
$$

Here, $\boldsymbol{\phi}_{-\mathrm{st}}$ denotes a random vector that includes every component of $\phi_{t}$ except for the sth component $\phi_{\mathrm{st}}, \operatorname{Normal}\left(\mu, \sigma^{2}\right)$ indicates the normal distribution with mean $\mu$ and variance $\sigma^{2}, w_{s^{\prime} s}$ is equal to 1 if counties $s$ and $s^{\prime}$ border each other (and is 0 otherwise), and $d_{s}$ is the number of counties that border county $s$. The parameter $\tau^{2}$ scales the variance structure of the CAR process. Note that the conditional variance of $\phi_{\mathrm{st}}$ is inversely proportional to the number of counties bordering it. Thus, if a county has more neighbors, its random effect has a smaller variance. This is desirable because if a county has many neighbors, there is more information to use to estimate its random effect. The parameter $\rho$ is also between 0 and 1 and controls the amount of correlation between bordering counties. As $\rho$ increases, so does the degree of dependence between the random effects in neighboring counties. It can be shown that the model specifications above define a valid joint distribution for $\phi_{t}$.

Our model specification is Bayesian in nature. To completely specify the model, prior distributions need to be assigned. The exact prior distributions used are listed in the previous forecasting publications (Bowman et al., 2016; Watson et al., 2017; Liu et al., $2017 a, 2017 b)$. Diffuse prior distributions were placed on the $\beta_{k}$ parameters. Diffuse priors have little to no influence on the posterior distribution, thus allowing the data to primarily drive inference and forecasting. Uninformative (flat) prior distributions are assigned to $\varphi$ and $\rho$ for the same reasons. Uninformative priors assign prior probability evenly over all feasible values of the parameter. The prior for $\tau^{2}$ is chosen as a conjugate prior, which means that the posterior and prior distributions are from the same distributional family, a computationally simplifying but reasonable assumption. A Markov chain Monte Carlo (MCMC) posterior sampling algorithm was developed to sample all model parameters and random effects from their posterior distributions. The $Y_{\text {st }}$ for counties not reporting test results were treated as latent (missing) variables and were sampled along with the unknown model parameters. For more on Bayesian models and MCMC methods, see Gelman et al. (2014).
To make a forecast, the model is first fit using all available test and covariate data for a fixed pathogen. The significance of covariate factors (whether or not they should be in the model) is judged using 95\% Bayesian credible intervals. All insignificant variables were removed resulting in a reduced model consisting of only significant variables; for specific details on how this was done, see Bowman et al. (2016), Liu et al. (2017a, 2017b), and Watson et al. (2017).

Once the model has been fit, all significant covariates and spatio-temporal random effects are forecasted for the forthcoming year. Forestation, water coverage, mosquito species presence/ absence, and elevation are assumed constant from year to year and do not need to be forecasted. These covariates are updated occasionally (e.g. when a new National Land Cover Database is released). Thus, the covariates that need to be forecasted for each county are annual temperature, annual relative humidity, annual precipitation, median household income, and population density. How this is done is described in Bowman et al. (2016), Liu et al. (2017a, 2017b), and Watson et al. (2017).

To forecast the spatial and temporal random effects a year in advance, Equation (3) is used. The $\boldsymbol{\phi}_{t}$ 's are independent and identically distributed over time given $\tau^{2}$ and $\rho$, which are sampled from the posterior distribution. A realization of $\phi_{t+1}$ is generated randomly from the fitted CAR model structure; one next sets $\boldsymbol{\xi}_{t+1}=\varphi \boldsymbol{\xi}_{t}+\boldsymbol{\phi}_{t+1}$. This process is repeated for each quartet of $\xi_{t}, \tau^{2}, \rho, \varphi$ from the posterior sample, thus yielding a sample of the forthcoming year's random effects. For further details, see Harrison and West (1996) and Gelman et al. (2014). A posterior sample of next year's forecasted prevalence, $p_{s, t+1}$, is then calculated from (2) using the posterior sample of the $\beta_{k}$ 's and the forecasted values of $X_{s, k, t+1}$ and $\xi_{s, t+1}$. Point estimates of the forecasted prevalence can be generated by taking the mean or median of the posterior sample of next year's prevalence.

Weighted correlation will be used to assess the accuracy of the forecasting methods. To compare the observed and forecasted county prevalence sets, a weighted correlation is computed. The contribution of each county to this correlation is weighted by the number of tests reported in that county; thus, counties not reporting data do not influence this correlation. The weighted correlation between two sets of county prevalences - say $A=$ $\left\{a_{1}, a_{2}, \ldots, a_{S}\right\}$ and $B=\left\{b_{1}, b_{2}, \ldots, b_{S}\right)-$ is defined as

$$
\operatorname{Corr}(A, B)=\frac{\sum_{s=1}^{S} h_{s}\left(a_{s}-a\right)\left(b_{s}-b\right)}{\sqrt{\sum_{s=1}^{S} h_{s}\left(a_{s}-a\right)^{2} \sum_{s=1}^{S} h_{s}\left(b_{s}-b\right)^{2}}},
$$

where $h_{s}$ is the total number of tests reported in county $s$ and the weighted means are

$$
a=\frac{\sum_{s=1}^{S} h_{s} a_{s}}{\sum_{s=1}^{S} h_{s}} ; \quad b=\frac{\sum_{s=1}^{S} h_{s} b_{s}}{\sum_{s=1}^{S} h_{s}}
$$

The interpretation of a weighted correlation is identical to that of the usual correlation, except that it accounts for unequal sample sizes through the weights. In particular, the more tests a county reports, the stronger the penalty is for deviations between the forecasted and observed prevalences. To create a final forecast map, the forecasted county prevalences are smoothed using Kriging, an interpolation method (Stein, 2012), using the default settings within ArcGIS (ESRI, 2016). 


\section{Forecasting fidelity assessment}

To measure forecasting accuracy in 2018 , forecasts for each pathogen were made from 2012-2017 data. The forecasts were then compared to the observed 2018 data via a weighted correlation as described in the preceding section. The same process has been completed for the 2016 and 2017 forecasts. Table 1 displays weighted correlations for each pathogen. Heartworm prevalence was the most difficult to predict and likely attributable to the complexity of factors regulating mosquito populations (Jian et al., 2014), but still had a correlation of around 0.78 . The weighted correlation for the three tick-borne pathogens exceeded 0.98 .

\section{Forecasts}

This section presents forecasts for 2019. Some of the covariate sources have changed from those making the 2016 and 2017 forecasts. For ease of interpretation, the 2019 forecasts are presented in the form of spatial prevalence maps. At the conclusion of 2019, data from this year will be used to compute correlations akin to those in Table 1 and assess the accuracy of the forecast.

Figure 1 shows the raw $B$. burgdorferi prevalence aggregated by county from 2012 to 2018 and the forecasted B. burgdorferi prevalence for 2019. Areas in the Appalachian region, northwestern Minnesota, and Indiana are expected to have higher than the average prevalence in 2019. The Atlantic coast, New England states, central Wisconsin, and southeastern Minnesota are all expected to see lower than average seroprevalence.

Figure 2 shows the raw Anaplasma spp. prevalence aggregated by county from 2012 to 2018, and the forecasted Anaplasma spp. prevalence for 2019. Greater than normal prevalence is predicted in New York and Pennsylvania, while areas along the Atlantic coast, in the upper Midwest, and southern Texas are expected to have lower prevalence.

Figure 3 shows the raw Ehrlichia spp. prevalence aggregated by county from 2012 to 2018, and the forecasted Ehrlichia spp. prevalence for 2019. Prevalence is predicted to be higher than average in the south-central US, particularly Oklahoma, Arkansas, and Missouri. Areas that are predicted to have a lower than average prevalence include eastern Arkansas and around the border of North Carolina and Virginia.

Figure 4 shows the raw heartworm prevalence aggregated by county from 2012 to 2018 , and the forecasted heartworm prevalence for 2019. Canine heartworm infection prevalence is expected to be higher than average throughout the southcentral and southeastern states. Areas of greatest concern are along the Mississippi River. Also expected to see higher prevalence are areas in Indiana, Illinois, and Iowa. A few small areas may see lower than average prevalence: southern Louisiana and southern Texas.

\section{Interpretation of forecast maps}

Prevalence is interpreted differently for different tests. As mentioned above, only $D$. immitis prevalence is based on antigentesting. Positive results from heartworm tests are more likely due to active infections; hence, the reported prevalence is close to a yearly incidence (rate of new cases in the population within a given time period). Elaborating, many positive dogs under the care of a veterinarian will subsequently test negative the following year; thus, dogs testing positive likely represent new cases. Despite this, the same dog could get multiple tests in a year. Without knowing treatment and testing history, annual prevalence cannot be considered as the true annual incidence. In contrast, tests for the three tick-borne pathogens are antibody-based. Typically, a seropositive dog has been exposed to the pathogen and has developed an antibody response; however, the presence of antibodies does not imply an active infection. Therefore, the population of seropositive animals for each of the tick-borne pathogens will consist of a mixture of previously exposed and uninfected animals, infected and subclinical animals, and infected and clinically ill animals. In short, the seroprevalence reported in the maps can be interpreted as the risk of exposure to each of the VBD pathogens.

Examination of the maps may reveal areas that appear anomalous above and beyond the aforementioned dynamic changes. For example, areas of unexpectedly high B. burgdorferi prevalence occur in some Rocky Mountain states. There are several issues to consider here, including data sample sizes, traveling dogs, a sicker 'testing population', and the presence of novel pathogens or vectors. Using Lyme disease and the Rocky Mountain states as an example, fewer tests are reported from this region. Hence, forecasts here have increased variability. To the authors' knowledge, there is no evidence of sustained enzootic transmission of B. burgdorferi sensu stricto in most of the Rocky Mountain states of New Mexico, Colorado, Utah, Wyoming, Idaho, and Montana. This said, traveling dogs that were exposed elsewhere have been linked to positive samples in this region (Millen et al., 2013). Dogs traveling with their owners to endemic regions are at risk of exposure and infection and may later test positive in a non-endemic county. This highlights the need to discuss pets' travel histories and future travel plans. In addition, some shelter animals are transported to different states for adoption purposes, or during disasters, such as Hurricane Katrina (Levy et al., 2011). These animals may be exposed prior to transport, and subsequently test positive in a non-endemic region. In light of these large movements of dogs and climate change, the possibility of both autochthonous and enzootic transmission should be considered. Dogs traveling with their families and those transported for adoption could create focal reservoirs that under ideal conditions could become sources for transmission (Morchón et al., 2012). Additionally, anomalous areas should not be outright discounted as inaccurate or unrepresentative of the local disease risk because the introduction of novel competent vectors or novel pathogens that cross-react on serologic tests may occur. An example of a novel pathogen observed in the data (E. muris eauclarensis) is discussed below. Prevalence may also be higher than expected if testing is biased towards animals with clinical signs of disease. Although the testing practices of veterinarians throughout the US is hard to quantify, practitioners in non-endemic regions may only test sick dogs, and may not routinely conduct wellness screening. If this occurs commonly within a region, then prevalence estimates would be skewed higher.

\section{Prevalence and forecast maps in practice}

\section{Evidence-based preventive medicine}

The cornerstone of companion animal general practice is preventive medicine. Annual wellness examinations are recommended by most veterinarians to address a multitude of health concerns: nutrition, oral health, parasite control, vaccinations, and screening tests, etc. Administration of preventive medication, vaccines, and testing is based on the standards of practice and disease risk levels. 
Table 1. Forecast fidelity assessment

\begin{tabular}{llll}
\hline & \multicolumn{3}{c}{ Weighted correlation } \\
\cline { 2 - 4 } Pathogen & 2016 & 2017 & 2018 \\
\hline Borrelia burgdorferi & 0.9869 & 0.9817 & 0.9870 \\
\hline Anaplasma spp. & 0.9873 & 0.9926 & 0.9918 \\
\hline Ehrlichia spp. & 0.9801 & 0.9801 & 0.9803 \\
\hline Dirofilaria immitis & 0.8624 & 0.8499 & 0.7835 \\
\hline
\end{tabular}

The table displays the weighted correlation between the observed prevalence and forecasted prevalence. This weighted correlation is similar to ordinary correlation, but here the contribution of each county to the overall correlation is weighted by the number of tests that each county reports. Thus, counties which report many tests contribute more to the correlation than counties which report only a few tests, and counties which do not report tests do not contribute at all. For details on how the weighted correlation is calculated, see Watson et al. (2017).

Here, two methods are presented for assessing the exposure risk for four canine vector-borne pathogens. Prevalence maps provide a local, real-time quantification of risk, while annual forecasts allow for preventive care recommendations to be proactively strengthened. Historically, the management of emerging infectious diseases has been retroactive, with the response coming after the disease significantly affects the population. With forecasts, higher than normal prevalence can be a priori predicted, allowing veterinarians to modify their preventative care protocols as needed. The changes (often increased prevalence) between the baseline prevalence and the forecasted year serve to highlight the areas in which veterinarians should be alerted to a possible change in the risk of exposure to their patients. Slowly changing risk, as can be the case with $\mathrm{VBD}$, can be difficult to monitor year to year. The modeling techniques utilized by the forecasts are more reliable with respect to accurately detecting these changes when compared to examining the prevalence maps alone. Additionally, approximately one-third of the counties in the US do not currently have these test data reported. As part of the model-building, the missing data from these counties are interpolated using known data from surrounding counties. As a result, the forecast maps allow us to predict the risk in areas that would otherwise have no information.

Both prevalence and forecast maps aid risk communication with clients. Effective communication skills are critical for the education of clients when making recommendations for preventive care and are essential for strengthening the veterinarian-client bond (Lue et al., 2008). From the client perspective, a clear understanding of recommendations is essential as up to $30 \%$ of clients do not follow recommendations when they lack clarity on the necessity of the intervention (Lue et al., 2008). Communication can be improved by supplementing conversations with written instructions, prepared handouts, and other visual aids (Coe et al., 2008). Both the prevalence and forecast maps are available for this purpose in a simple format and on multiple platforms. The ability to display the maps on hand-held devices allows consultation of the maps in the exam room. Local prevalence statistics provide veterinarians an opportunity to engage clients in datadriven conversations about the risk of VBD.

Within the context of the veterinarian-client conversation, it is important to incorporate the pet's individual risk of exposure to vectors. Clients expect a personalized approach (Coe et al., 2008), which ensure that the recommended preventive care measures are appropriate for that pet. Veterinarians should ask whether the pet spends any time in favored tick habitats (areas with tall grass, leaf litter, woods, and brush), including areas around the client's home, and about past and future travel. Travel with pets, particularly dogs, has increased significantly in the last decade, with $37 \%$ of clients reporting pets accompany them on trips (American Pet Products Association, 2017). This is especially important when a client is traveling from a non-endemic to an endemic region. To help facilitate this, CAPC provides prevalence maps at the national, state, and county level for in-clinic communication efforts as the knowledge of vector-borne pathogens outside the home county may be limited for the veterinarian or client.

Unambiguous risk communication leads to a mutual understanding of an individual pet's risk. This allows veterinarians to further educate clients on why the proactive management of VBD is important. Preventative measures vary by the pathogen of concern and currently include annual testing with pet-side assays such as those for D. immits, Ehrlichia spp., Anaplasma spp., and B. burgdorferi, administration of acaricides to reduce the exposure to tick-borne pathogens, anthelmintics to prevent the development of $D$. immitis, and when appropriate, vaccination against $B$. burgdorferi. Surveys suggest that owners become confused about the value of services and products offered during wellness examination when offered a multitude of choices (Lue et al., 2008). If overwhelmed, clients usually choose fewer services. Explaining the value of services is cited as being perceived by clients as increasing the quality of care provided during annual wellness exams. In particular, communication about the cost-savings of preventing infection, early detection of disease, prevention of long-term health complications, and early treatment become invaluable in fostering the veterinarian-client relationship. Average costs range from $\$ 215$ (US\$ 2017) (Nationwide Insurance, personal communication) to $\$ 1000$ (2017 US\$) (Bowman and Atkins, 2009; Colby et al., 2011; Maxwell et al., 2014; Wolstenholme et al., 2015) depending on the infection; however, not all animals that test positive for the various pathogens are treated. In the case of anaplasmosis, the majority of seropositive dogs are subclinical. Therefore, current recommendations for veterinary care in Anaplasma spp. seroreactive dogs include a complete blood count with platelet count to determine if treatment is necessary (Little, 2010).

Unfortunately, no consolidated reports exist regarding the industry-level annual financial impact of VBD in companion animals. As a result, the financial burden of VBD is difficult to estimate. Lyme disease treatment is approximately $\$ 215$ per pet (Nationwide Insurance, personal communication). When aggregated across the veterinary healthcare system, the annual cost likely lies somewhere between $\$ 3.3$ and $\$ 54$ million, with the low estimate representing the percentage of seropositive animals that were treated based on insurance claims (Nationwide Insurance, personal communication), and the high estimate representing the treatment of all seropositive animals. Similar estimates can be made for the rickettsial diseases, with both ehrlichiosis and anaplasmosis having average treatment costs of $\$ 221$ per patient (Nationwide Insurance, personal communication). From this, treatment for ehrlichiosis is estimated to range from $\$ 1.1$ to $\$ 23$ million, and anaplasmosis from $\$ 1.1$ and $\$ 25$ million. Finally, the cost for treatment for heartworm infection is by far the most significant, averaging $\$ 400-\$ 1000$ per patient (Bowman and Atkins, 2009; Colby et al., 2011; Maxwell et al., 2014; Wolstenholme et al., 2015) and costing US pet owners approximately $\$ 75$ million annually. Collectively, the burden of 
VBD on the veterinary health care sector is estimated to be $\$ 80.5-$ \$177 million annually, and the costs of treatment may change over time. Note that these estimates are based on just one of many insurance companies and may not represent out-of-pocket costs and those spent on complicated cases. The broad range of estimates for the financial impact of VBD highlights an opportunity for a subsequent analysis of cost that is inclusive of all stakeholders, including the pet insurance industry, corporate veterinary groups and regional private practices.

Despite our limitations in clearly defining financial impact, the financial burden of treating infected animals supports the need for anthelmintics, acaricides, vaccinations, and testing. For veterinarians, there is an obvious cost-to-benefit and financial saving to pet owners who opt for prevention, yet most veterinarians face challenges with preventive compliance. Administration of preventive medications is generally recommended year-round to prevent any exposure windows (Nelson et al., 2014; Companion Animal Parasite Council, 2018; US Food and Drug Administration, 2018); yet, the majority of dogs only receive seasonal treatment, averaging 6-8 months of heartworm prevention (Gates and Nolan, 2010). Only $64 \%$ of clients use parasiticides on a year-round basis (American Pet Products Association, 2017). Similarly, in a recent survey, only $62 \%$ of owners recalled their veterinarian's recommendation of 12-month coverage for flea and tick prevention (Lavan et al., 2017). The seasonal window for exposure risk depends on the phenology (seasonality) of the vectors, which is influenced by dynamic climatological factors (Eisen et al., 2015) and can vary temporally and geographically, even within a small region of a state. Attempting to predict this window and only provide prevention could result in inadvertent exposure that could have been easily prevented with year-round administration.

Beyond preventive treatments, veterinarians practicing in areas of high $B$. burgdorferi seroprevalence may also recommend vaccination against $B$. burgdorferi. The maps are useful in identifying endemic regions of Lyme disease to determine when a Lyme disease vaccine should be recommended, with the additional consideration of having a discussion with clients about frequent travel with pets to well-established endemic regions such as the Northeast and Central Upper Midwest. Veterinarians in regions between high- and low-prevalence areas could use the prevalence and forecast maps in conjunction with knowledge of the individual dog to establish a cost-to-benefit of vaccination. Guidelines for the prevention and treatment of VBD and a broad range of parasitic diseases are accessible at http://www.capcvet.org/guidelines/, recent guidelines from the American College of Veterinary Internal Medicine on Lyme borreliosis in dogs are available (Littman et al., 2018), and guidelines on the prevention, diagnosis, and treatment of heartworm are available from the American Heartworm Society (Nelson et al., 2014). Despite the availability of preventative medications and vaccines, pets are never $100 \%$ protected against exposure (Vogt et al., 2019). Therefore, annual screening tests are recommended (Nelson et al., 2014; Companion Animal Parasite Council, 2018; US Food and Drug Administration, 2018). Furthermore, additional testing data improve the fidelity of the forecasts. Reporting of test results back to the diagnostic company greatly aids VBD surveillance, particularly when in-clinic tests are utilized.

Finally, most practitioners recognize a shift in veterinary-client interactions toward a consumer-centric model where clients are frequently empowered consumers and prefer to have a voice in their pet's healthcare decisions. Pet owners often enter the exam room armed with information and opinions on optimal care for their pets. Sometimes, clients' preconceived ideas are at odds with the practitioner's training and knowledge base, with the source of information used by clients often coming from Internet sources of questionable validity. To satisfy growing consumer demands for information, VBD forecast and prevalence maps are available at the CAPC consumer-focused website www.petsandparasites.org. These maps provide accurate information on disease prevalence, with an emphasis that their veterinarian is the local expert on VBD testing, prevention, and treatment. Communication of science to the general public is increasingly recognized as a core responsibility of scientists and health care providers; ultimately, the presentation of accurate information in jargonless language allows clients to make informed decisions about prevention measures.

\section{One Health}

Often, the VBD conversation between veterinarians and clients extends to the human risk and the health and well-being of clients and their family. Many vector-borne pathogens infect both people and dogs, including B. burgdorferi, E. chaffeensis, E. ewingii, A. phagocytophilum, and $A$. platys. A recent study found that tick-infested dogs can transfer ticks directly to human hosts during interactions and serve as transport hosts, carrying ticks in and around dwellings (Jones et al., 2018). For these zoonotic pathogens, the CVBD maps suggest where human beings are at risk of exposure (Liu et al., 2019). Unfortunately, routine testing is not conducted for many other vector-borne pathogens of relevance to people and dogs and general surveillance is lacking. However, one may still derive pathogen risk information when a vector of one of the mapped pathogens is shared. For example, co-infections of Ixodes scapularis with multiple pathogens (e.g. B. burgdorferi, Borrelia miyamotoi, A. phagocytophilum, Babesia microti, and Bartonella spp.) are common (Adelson et al., 2004; Diuk-Wasser et al., 2016), and infection with Ehrlichia species and several other vector-borne pathogens such as Rickettsia and Bartonella have been concurrently observed in people and dogs (Kordick et al., 1999). Wildlife is the reservoir for many vectorborne pathogens and the environment is a critical factor in determining the emergence of those pathogens (Daszak et al., 2001), highlighting the incredible importance of One Health, not just public health, in the prevention of VBD in all hosts. Inclusion of information about family risk serves to foster client relationships and illustrates the professional commitment of veterinary practitioners to pets and their owners.

\section{Vector-borne disease research}

Vector information, such as endemic regions and abundance, are critical for understanding VBD establishment and spread. Unfortunately, accurate data on vector populations are currently economically and logistically unfeasible to collect, and such data deficiencies will likely continue to limit micro-scale VBD knowledge. Given the logistical and financial barriers, targeting vectorspecific research to emerging areas generally has the greatest impact. Forecast estimates can be used to guide study designs for regional comparison of vector presence and abundance, comparative studies in zoonotic disease research relating human incidence to companion animal disease prevalence, and VBD research comparing different canine populations. 
Examples of the impact of VBD mapping on research are evident in the literature. For example, an annual analysis of VBD has revealed regional foci of infections that were inexplicable in the context of what was currently known about the pathogens or present vectors. Specifically, in 2009, researchers were surprised by the foci of seroreactivity to Ehrlichia spp. in dogs in Wisconsin and Minnesota, a region where E. chaffeensis is not present or is only present in low prevalence (Bowman et al., 2009). Investigators reasoned that there may be foci of intense E. canis transmission in these specific regions, or alternately, exposure of dogs to a novel Ehrlichia spp. that has yet to be described. Soon after, researchers identified four human cases of ehrlichiosis in Minnesota and Wisconsin that were not caused by E. chaffeensis or E. ewingii, but instead by a newly discovered Ehrlichia species, ultimately named E. muris euclairensis (Pritt et al., 2011). By 2012, researchers identified a dog infected with E. muris euclairensis in the same region (Hegarty et al., 2012), illustrating that anomalies can be early indicators of an emerging disease. In another example, unexpected increases in Anaplasma spp. seroprevalence have been observed in Texas, notably on the Mexican border and along the western edge of the state. These observations are notable given the recent study by Movilla et al., who documented canine seroprevalence of Anaplasma spp. in several states in Mexico (Movilla et al., 2016) (the highest seroprevalence occurs in northwestern Mexico at $16.4 \%$, and the lowest occurs in the north-central states of the country at $0.6 \%$ ).

It is important for map users to understand that the estimates shown in the maps are based on historical data. As such, rapidly occurring events are unlikely to be predicted. This is less of a concern with tick-borne disease, which generally exhibits slow spread and is not prone to rapid, large-scale epidemics. However, forecast models of tick-borne pathogens may be improved by the inclusion of predictors shown to forecast the distribution and densities of ticks (Feria et al., 2014; Brugger et al., 2018). In contrast, mosquito vectors of $D$. immitis may be strongly influenced by shortterm disturbances in weather patterns, and notably in abundance of bodies of water, both standing and transitory (Valle et al., 2013). Until a better understanding of the ecology of mosquitoborne disease transmission at a fine spatial and temporal scale is achieved, it will be difficult to forecast short-term changes.

There is still much to learn about the epidemiology of canine vector-borne infections. Novel pathogens are continually being discovered in dogs and people. Additional organisms are detected in wildlife species that could one day emerge in other hosts. These new pathogens pose diagnostic challenges and generally are poorly understood in regard to vector(s) used, reservoir host(s) of importance, and clinical disease risks. Regarding modeling, future studies are needed to produce long-term forecasts, develop models that can define areas that are experiencing increasing/ decreasing prevalence in both space and time, understand the impact of natural disasters on prevalence, understand the prevalence of disease, not just exposure, within the population, and ultimately evaluate the application of these data to forecast human exposure risk. Canadian testing data has been recently made available and future studies will seek to include these data. Veterinarians in Canada use similar testing practices, allowing us to expand the forecasts to include Canada.

\section{Conclusion}

The prevalence and forecast maps reviewed here are designed to be a timely aid for veterinary practitioners in developing preventive care protocols by keeping veterinarians up to date on the present risk of exposure to several vector-borne pathogens. The maps allow veterinarians to practice evidence-based medicine and help in risk communication with pet owners. In particular, the forecast maps allow veterinarians to be proactive in their prevention protocols by giving accurate estimates to the expected level of local risk for the coming year.

Acknowledgments. IDEXX Laboratories, Inc. and Antech Diagnostics are thanked for their data contributions. The authors also thank Dr Carol McConnell, Chief Veterinary Officer, Nationwide, for providing VBD claims data that enabled the calculation of the financial impact of the disease. The Companion Animal Parasite Council financially supported this work. YL and RBL thank the National Science Foundation Grant DMS 1407480. CSM was partially supported by the National Science Foundation grant OIA-1826715. JRG is supported by The Boehringer Ingelheim Vetmedica-CAPC Infectious Disease Postdoctoral Fellowship.

\section{References}

Adelson ME, Rao RV, Tilton RC, Cabets K, Eskow E, Fein L, Occi JL and Mordechai E (2004) Prevalence of Borrelia burgdorferi, Bartonella spp., Babesia microti, and Anaplasma phagocytophila in Ixodes scapularis ticks collected in Northern New Jersey. Journal of Clinical Microbiology 42 2799-2801.

Allison RW and Little SE (2013) Diagnosis of rickettsial diseases in dogs and cats. Veterinary Clinical Pathology 42, 127-144.

American Pet Products Association (2017) 2017-2018 National Pet Owners Survey.

Atkins CE (2003) Comparison of results of three commercial heartworm antigen test kits in dogs with low heartworm burdens. Journal of the American Veterinary Medical Association 222, 1221-1223.

Banerjee S, Carlin BP and Gelfand AE (2014) Hierarchical Modeling and Analysis for Spatial Data, 2nd Edn. Boca Raton: Chapman \& Hall/CRC.

Besag J (1974) Spatial interaction and the statistical analysis of lattice systems. Journal of the Royal Statistical Society. Series B (Methodological) 36, 192-236.

Bowman DD (2014) Helminths. In Bowman DD (ed.), Georgis' Parasitology for Veterinarians, 10th Edn. St. Louis: Elsevier Health Sciences, pp. $212-$ 221.

Bowman DD and Atkins CE (2009) Heartworm biology, treatment, and control. The Veterinary Clinics of North America. Small Animal Practice 39, 1127-1158.

Bowman D, Little SE, Lorentzen L, Shields J, Sullivan MP and Carlin EP (2009) Prevalence and geographic distribution of Dirofilaria immitis, Borrelia burgdorferi, Ehrlichia canis, and Anaplasma phagocytophilum in dogs in the United States: results of a national clinic-based serologic survey. Veterinary Parasitology 160, 138-148.

Bowman DD, Liu Y, McMahan CS, Nordone SK, Yabsley MJ and Lund RB (2016) Forecasting united states heartworm Dirofilaria immitis prevalence in dogs. Parasites \& Vectors 9, 540.

Brockwell PJ and Davis RA (2002) Introduction to Time Series and Forecasting, 2nd Edn. New York City: Springer-Verlag.

Brugger K, Walter M, Chitimia-Dobler L, Dobler G and Rubel F (2018) Forecasting next season's Ixodes ricinus nymphal density: the example of southern Germany 2018. Experimental and Applied Acarology 75, 281-288.

Coe JB, Adams CL and Bonnett BN (2008) A focus group study of veterinarians' and pet owners' perceptions of veterinarian-client communication in companion animal practice. Journal of the American Veterinary Medical Association 233, 1072-1080.

Colby KN, Levy JK, Dunn KF and Michaud RI (2011) Diagnostic, treatment, and prevention protocols for canine heartworm infection in animal sheltering agencies. Veterinary Parasitology 176, 333-341.

Companion Animal Parasite Council (2018) Companion Animal Parasite Council. https://www.capcvet.org (Accessed 11 November 2017).

Daszak P, Cunningham AA and Hyatt AD (2001). Anthropogenic environmental change and the emergence of infectious diseases in wildlife. Acta Tropica 78:103-116. 
Diuk-Wasser MA, Vannier E and Krause PJ (2016) Coinfection by Ixodes tick-borne pathogens: ecological, epidemiological, and clinical consequences. Trends in Parasitology 32, 30-42.

Eisen RJ, Eisen L, Ogden NH and Beard CB (2015) Linkages of weather and climate with Ixodes scapularis and Ixodes pacificus (Acari: Ixodidae), enzootic transmission of Borrelia burgdorferi, and Lyme disease in North America. Journal of Medical Entomology 53, 250-261.

ESRI (Environmental Systems Research Institute) (2016) http://desktop.arcgis.com/en/documentation/ (Accessed 10 November 2017).

Feria-Arroyo TP, Castro-Arellano I, Gordillo-Perez G, Cavazos AL, Vargas-Sandoval M, Grover A, Torres J, Medina RF, de León AA and Esteve-Gassent MD (2014) Implications of climate change on the distribution of the tick vector Ixodes scapularis and risk for Lyme disease in the Texas-Mexico transboundary region. Parasites \& Vectors 7, 199.

Gates MC and Nolan TJ (2010) Factors influencing heartworm, flea, and tick preventative use in patients presenting to a veterinary teaching hospital. Preventive Veterinary Medicine 93, 193-200.

Gelman A, Carlin JB, Stern HS and Rubin DB (2014) Bayesian Data Analysis, 3rd Edn. Boca Raton, FL: Chapman and Hall/CRC.

Greene CE (2012) Infectious Diseases of the Dog and Cat, 4th Edn. St. Louis, MO: Elsevier Health Sciences.

Harrison J and West M (1996) Bayesian Forecasting and Dynamic Models, 2nd Edn. New York City: Springer.

Hegarty B, Maggi R, Koskinen P, Beall M, Eberts M, Chandrashekar R and Breitschwerdt E (2012) Ehrlichia muris infection in a dog from Minnesota. Journal Veterinary Internal Medicine 26, 1217-1220.

Jian Y, Silvestri S, Brown J, Hickman R and Marani M (2014) The temporal spectrum of adult mosquito population fluctuations: conceptual and modeling implications. PLoS ONE 9, e114301.

Jones E, Hinckley A, Hook S, Meek J, Backenson B, Kugeler K and Feldman K (2018) Pet ownership increases human risk of encountering ticks. Zoonoses and Public Health 65, 74-79.

Kordick SK, Breitschwerdt EB, Hegarty BC, Southwick KL, Colitz CM, Hancock SI, Bradley JM, Rumbough R, Mcpherson JT and MacCormack JN (1999) Coinfection with multiple tick-borne pathogens in a Walker Hound kennel in North Carolina. Journal of Clinical Microbiology 37, 2631-2638.

Lavan RP, Tunceli K, Zhang D, Normile D and Armstrong R (2017) Assessment of dog owner adherence to veterinarians' flea and tick prevention recommendations in the United States using a cross-sectional survey. Parasites \& Vectors 10, 284.

Levy JK, Lappin MR, Glaser AL, Birkenheuer AJ, Anderson TC and Edinboro CH (2011) Prevalence of infectious diseases in cats and dogs rescued following hurricane Katrina. Journal of the American Veterinary Medical Association 238, 311-317.

Little SE (2010) Ehrlichiosis and anaplasmosis in dogs and cats. The Veterinary Clinics of North America. Small Animal Practice 40, 1121-1140.

Little SE, Heise SR, Blagburn BL, Callister SM and Mead PS (2010) Lyme borreliosis in dogs and humans in the USA. Trends in Parasitology 26, 213-218.

Littman MP, Gerber B, Goldstein RE, Labato MA, Lappin MR and Moore GE (2018) ACVIM consensus update on Lyme borreliosis in dogs and cats. Journal of Veterinary Internal Medicine 32, 1-17.

Liu Y, Lund RB, Nordone SK, Yabsley MJ and McMahan CS (2017a) A Bayesian spatio-temporal model for forecasting the prevalence of antibodies to Ehrlichia species in domestic dogs within the contiguous United States. Parasites \& Vectors 10, 138.

Liu Y, Watson SC, Gettings JR, Lund RB, Nordone SK, Yabsley MJ and McMahan CS (2017b) A Bayesian spatio-temporal model for forecasting Anaplasma species seroprevalence in domestic dogs within the contiguous United States. PLoS ONE 12, e0182028.

Liu Y, Nordone SK, Yabsley MJ, Lund RB, McMahan CS and Gettings JR (2019) Quantifying the relationship between human Lyme disease and Borrelia burgdorferi exposure in domestic dogs. Geospatial Health. doi: 10.13140/RG.2.2.20689.99689.

Loh EH, Zambrana-Torrelio C, Olival KJ, Bogich TL, Johnson CK, Mazet JA, Karesh W and Daszak P (2015) Targeting transmission pathways for emerging zoonotic disease surveillance and control. Vector-borne and Zoonotic Diseases 15, 432-437.

López-Quilez A and Munoz F (2009) Review of spatio-temporal models for disease mapping. Final Report for the EUROHEIS, 2.

Lue TW, Pantenburg DP and Crawford PM (2008) Impact of the owner-pet and client-veterinarian bond on the care that pets receive. Journal of the American Veterinary Medical Association 232, 531-540.

Maggi RG, Birkenheuer AJ, Hegarty BC, Bradley JM, Levy MG and Breitschwerdt EB (2014) Comparison of serological and molecular panels for diagnosis of vector-borne diseases in dogs. Parasites \& Vectors 7, 127.

Martínez-Beneito MA, López-Quilez A and Botella-Rocamora P (2008) An autoregressive approach to spatio-temporal disease mapping. Statistics in Medicine 27, 2874-2889.

Maxwell E, Ryan K, Reynolds C and Pariaut R (2014) Outcome of a heartworm treatment protocol in dogs presenting to Louisiana State University from 2008 to 2011: 50 cases. Veterinary Parasitology 206, 71-77.

Mead PS (2015) Epidemiology of Lyme disease. Infectious Disease Clinics of North America 29, 187-210.

Millen K, Kugeler KJ, Hinckley AF, Lawaczeck EW and Mead PS (2013) Elevated Lyme disease seroprevalence among dogs in a nonendemic county: Harbinger or artifact. Vector-borne and Zoonotic Diseases 13, 340-341.

Morchón R, Carretón E, González Miguel J and Mellado Hernández I (2012) Heartworm disease (Dirofilaria immitis) and their vectors in Europe - new distribution trends. Frontiers in Physiology 3, 196.

Movilla R, García C, Siebert S and Roura X (2016) Countrywide serological evaluation of canine prevalence for Anaplasma spp., Borrelia burgdorferi (sensu lato), Dirofilaria immitis and Ehrlichia canis in Mexico. Parasites \& Vectors 9, 421

National Notifiable Diseases Surveillance System (NNDSS), Centers for Disease Control and Prevention. https://wwwn.cdc.gov/nndss/ (Accessed 5 January 2018).

Nelson CT, McCall JW and Carithers D (2014) Current Canine Guidelines for the Prevention, Diagnosis, and Management of Heartworm (Dirofilaria immitis) Infection in Dogs. Wilmington: American Heartworm Society, pp. 1-22.

Pritt BS, Sloan LM, Johnson DK, Munderloh UG, Paskewitz SM, McElroy KM, McFadden JD, Binnicker MJ, Neitzel DF, Liu G, Nicholson WL, Nelson CM, Franson JJ, Martin SA, Cunningham SA, Steward CR, Bogumill K, Bjorgaard ME, Davis JP, McQuiston JH, Warshauer DM, Wilhelm MP, Patel R, Trivedi VA and Eremeeva ME (2011) Emergence of a new pathogenic Ehrlichia species, Wisconsin and Minnesota, 2009. The New England Journal of Medicine 365, 422-429.

Qurollo BA, Chandrashekar R, Hegarty BC, Beall MJ, Stillman BA, Liu J, Thatcher B, Pultorak E, Cerrito B, Walsh M and Breitschwerdt EB (2014) A serological survey of tick-borne pathogens in dogs in North America and the Caribbean as assessed by Anaplasma phagocytophilum, A. platys, Ehrlichia canis, E. chaffeensis, E. ewingii, and Borrelia burgdorferi speciesspecific peptides. Infection Ecology \& Epidemiology 4, 24699.

Rosenberg R, Lindsey NP, Fischer M, Gregory CJ, Hinckley AF, Mead PS, Paz-Bailey G, Waterman SH, Drexler NA, Kersh GJ and Hooks H (2018) Vital signs: trends in reported vectorborne disease cases - United States and Territories, 2004-2016. Morbidity and Mortality Weekly Report 67, 496.

Stein ML (2012) Interpolation of Spatial Data: Some Theory for Kriging. New York: Springer Science \& Business Media.

Sykes JE (2014) Canine and Feline Infectious Diseases. St. Louis: Elsevier Health Sciences.

Taylor LH, Latham SM and Mark E (2001) Risk factors for human disease emergence. Philosophical Transactions of the Royal Society of London. Series B, Biological Sciences 356, 983-989.

United States Food and Drug Administration (2018) Prevent heartworms in dogs, cats, and ferrets year-round. https://www.fda.gov/ForConsumers/ ConsumerUpdates/ucm371377.htm (Accessed 3 April 2019).

Valle D, Zaitchik B, Feingold B, Spangler K and Pan W (2013) Abundance of water bodies is critical to guide mosquito larval control interventions and predict risk of mosquito-borne diseases. Parasites \& Vectors 6, 179.

Vogt NA, Sargeant JM, MacKinnon MC and Versluis AM (2019) Efficacy of Borrelia burgdorferi vaccine in dogs in North America: a systematic review and meta-analysis. Journal of Veterinary Internal Medicine 33, 23-36. 
Wang D, Bowman DD, Brown HE, Harrington LC, Kaufman PE, McKay T, Nelson CT, Sharp JL and Lund R (2014) Factors influencing US canine heartworm (Dirofilaria immitis) prevalence. Parasites \& Vectors 7, 264.

Watson SC, Liu Y, Lund RB, Gettings JR, Nordone SK, McMahan CS and Yabsley MJ (2017) A Bayesian spatio-temporal model for forecasting the prevalence of antibodies to Borrelia burgdorferi, causative agent of Lyme disease, in domestic dogs within the contiguous United States. PLOS ONE 12, $\mathrm{e} 0174428$.

Wolstenholme AJ, Evans CC, Jimenez PD and Moorhead AR (2015) The emergence of macrocyclic lactone resistance in the canine heartworm, Dirofilaria immitis. Parasitology 142, 1249-1259.

Woolhouse ME and Gowtage-Sequeria S (2005) Host range and emerging and reemerging pathogens. Emerging Infectious Diseases 11, 1842. 